\title{
Coverage Prediction and Performance Evaluation of Wireless Metropolitan Area Networks based on IEEE 802.16
}

\author{
Fabricio Lira Figueiredo, and Paulo Cardieri, Member, IEEE
}

\begin{abstract}
This work aims at performing a more detailed coverage and performance analysis of wireless metropolitan area networks based on the IEEE 802.16 standard, which is the basis of WiMAX technology. This paper provides a more formal approach to estimate coverage and performance of WiMAX technology, which enables simplified link budget evaluation. Initially, the major functionalities within the standard that support operation in NLOS scenarios are described. Several operation scenarios are then specified, based on the combination of key system parameters (e.g., transmission power, operation frequency, channel bandwidth) and coverage prediction models. In order to support coverage estimation for operation frequencies up to $6 \mathrm{GHz}$, appropriate coverage prediction models are adopted for frequencies above $2 \mathrm{GHz}$. Based on the proposed approach, coverage and performance are estimated under some real world scenario conditions, considering Brazilian regulatory rules for licensed and license-exempt bands.
\end{abstract}

Index Terms-WiMAX, IEEE 802.16, wireless metropolitan area, coverage, performance, propagation models.

\section{INTRODUCTION}

$\mathbf{R}$ ECENTLY, some novel wireless technologies have been developed by the IEEE 802 Working Groups, in collaboration with the industry, with focus on interoperability, flexibility, low cost, all-IP support and high transmission data rate. Wi-Fi technology is an example of such approach. It is a widely adopted wireless LAN solution, specified by the IEEE 802.11 standard [1]. It supports operation in license-exempt frequencies (2.4 and $5.8 \mathrm{GHz}$, in Brazil), with coverage radius up to hundreds of meters and transmission rate up to $54 \mathrm{Mbps}$.

Following the same strategy, the IEEE 802 Working Group has been specifying the IEEE 802.16 standard [2], which describes the basis of WiMAX technology. It consists of a fixed wireless metropolitan area technology that supports coverage radius of kilometers and data transmission rate up to 74 Mbps. Furthermore, it supports QoS on the wireless domain and interfaces for E1/T1, ATM, IP and Ethernet. Due to the functionalities supported by WiMAX technology, several broadband services can be deployed, including Voice over IP and video on-demand.

In this context, it is worth mentioning the role played by WiMAX Forum. The major purpose of this forum is to assure interoperability and standard compliance of the equipments from different vendors, which is a key success factor for any

Fabricio Lira Figueiredo, CPqD Telecom and IT Solutions, Campinas, Brazil, e-mail: fabricio@cpqd.com.br; Paulo Cardieri, State University of Campinas, UNICAMP, Brazil, e-mail: cardieri@decom.fee.unicamp.br.

This work was performed at $\mathrm{CPqD}$ Telecom and IT Solutions. modern telecommunication technology. Furthermore, WiMAX Forum handles regulatory issues around the world and is essentially composed of WiMAX equipment and chipset manufacturers, as well as carriers and Internet Service Providers (ISP's).

Coverage prediction and performance evaluation for the IEEE 802.16 standard has not yet been deeply explored in the literature. Some coverage prediction models for systems beyond $3 \mathrm{G}$ have been analyzed based on the extrapolated versions of Hata and Walfisch-Ikegami models, with no specific focus on the IEEE 802.16 specifications [4]. A more detailed approach regarding WiMAX coverage is provided in [5], which briefly describes the key functionalities of WiMAX technology that enable NLOS operation and mentions Stanford University Interim model (SUI), providing a single example of operation scenario.

Despite the growing interest on WiMAX technology, detailed procedures for coverage prediction and performance evaluation of IEEE 802.16 networks are not yet clearly stated in the literature for general operation conditions, due to some specific limitations. The first one is related to the lack of currently available coverage prediction models that comply with operation frequencies requirements of WiMAX, especially for frequencies ranging from $2 \mathrm{GHz}$ up to $6 \mathrm{GHz}$. Secondly, the quantitative influence of system parameters on coverage is not clearly described within the IEEE 802.16 standard. This paper provides a more formal approach to evaluate coverage and performance of WiMAX technology, presenting equations that enable a simplified link budget evaluation, comprising the most appropriate coverage models currently available and the key system attributes defined in the IEEE 802.16 standard. Coverage and performance estimates are provided, based on real world operation, in accordance to the current Brazilian regulatory rules, as per [6] and [7], which respectively handles operation in license-exempt (2.4 and $5 \mathrm{GHz})$ and licensed (3.5 Ghz) bands.

The paper is organized as follows: Section II presents a brief description of the historical evolution of the IEEE 802.16 standard and the major aspects of the technology, such as network topology and architecture, protocol architecture, and some key functionalities for NLOS operation, in accordance to IEEE 802.16-2004 standard. Section III provides a description of the key elements for coverage and performance evaluation of WiMAX wireless networks, based on currently available and appropriate propagation models, as well as on analytical formulas for the OFDM receiver sensitivity and maximum data 


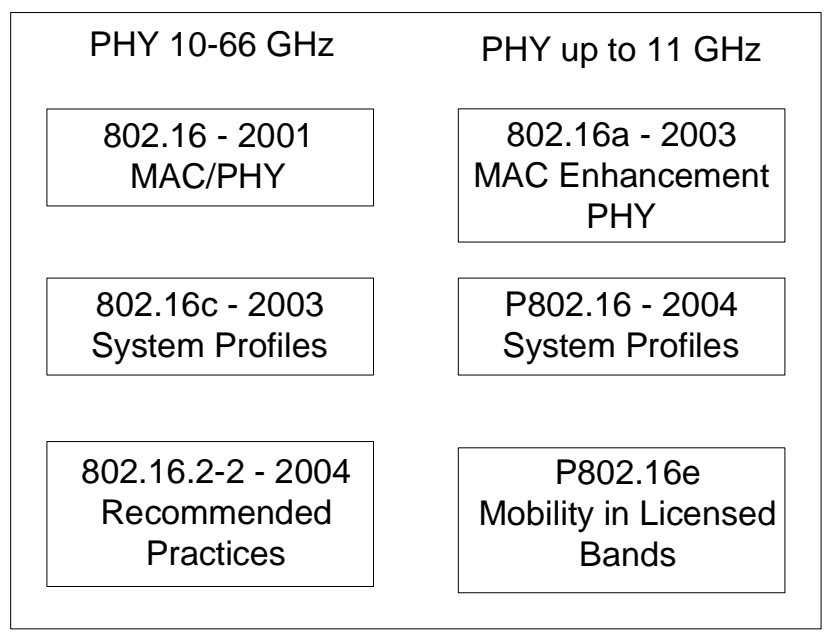

Fig. 1. Versions of IEEE 802.16 standards.

transmission rate, based on key system parameters defined in the standard. In Section IV, coverage prediction and performance are evaluated, based on licensed and license-exempt operation scenarios, which comprise system configurations optimized for throughput and coverage. Section V concludes de paper.

\section{IEEE 802.16 STANDARD}

The standards within the IEEE 802.16 family and their release dates are depicted in Figure 1. The first version was focused on LOS operation in the range from 10 to $66 \mathrm{GHz}$. In 2003, the version IEEE 802.16c was published, including recommendations for the system profiles for the 10$66 \mathrm{GHz}$ range, thus contributing to interoperability. In 2004, the 802.16.2-2 version was published, which corresponds to a set of best practices for deployment of IEEE 802.16 networks, in several real world scenarios.

However, LOS operation often represents a major limitation on the deployment of a wireless network. In order to overcome this limitation, the 802.16 a version was developed to include extensions of physical and medium access control layers, for operation in frequencies between 2 and $11 \mathrm{GHz}$. In 2004, the 802.16-2004 version was published, which corresponds to a consolidated specification for LOS and NLOS operation in frequencies up to $66 \mathrm{GHz}$ (including frequencies below 11 $\mathrm{GHz}$ ). This version has been adopted as the major standard for the development of the first generation of WiMAX chipsets and equipments.

Finally, the version $802.16 \mathrm{e}$ is under development and will incorporate functionalities to support mobility, thus allowing the WiMAX technology to be embedded in portable devices (laptops and handhelds).

\section{A. Network Topology and Architecture}

The network topology and architecture, as specified in the IEEE 802.16 standard [2], comprise Base Station (BS) and Subscriber Station (SS), which correspond to the basic network elements of WiMAX technology, as depicted in Figure 2. In

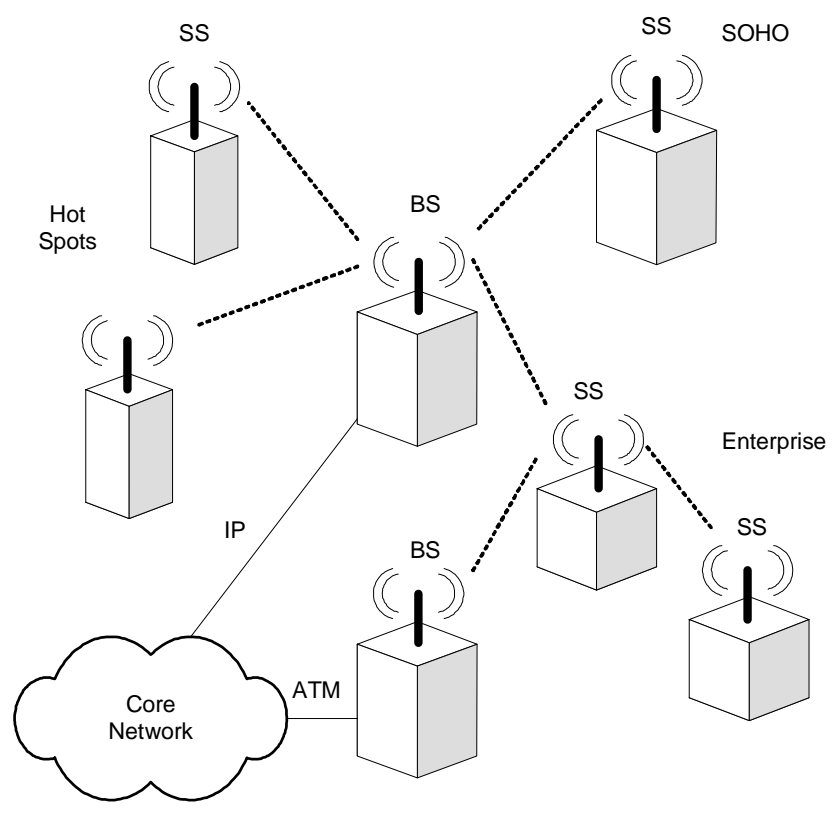

Fig. 2. Network topology and architecture.

the Point-to-Multipoint (PMP) topology, the BS coordinates the medium access and supports ATM, E1/T1, IP and Ethernet interfaces to the core network (IP backbone). The SS provides network access to the subscriber via a wireless link to the BS.

The standard also specifies Mesh topology as an optional functionality, as illustrated in Figure 2. In this case, one SS is allowed to setup wireless links directly to another SS, with no intermediate BS. This capability results in a wireless multihop networking solution, which is a quite flexible and cost-effective approach to increase coverage, since the number of BS's in a real world deployment can be significantly reduced.

\section{B. Protocol Architecture}

The current version of the IEEE 802.16 standard comprises the specification of physical (PHY) and medium access control (MAC) layers. The MAC layer performs centralized or distributed scheduling to control the medium access, thus preventing collisions of subscribers connected to the same BS. It also manages QoS in the wireless links domain, via dynamic bandwidth reservation and traffic priority handling mechanisms. Support to multiple interfaces to core network and physical layer versions, as well as synchronization and security mechanisms, also constitute key MAC layer functionalities.

The major purpose of the PHY layer is to properly process the raw bit information in order to minimize the errors at the receiver. In order to achieve the high performance levels required to support wireless broadband services, advanced modulation, equalization, multiplexing, diversity schemes and error control schemes are specified. The multiple versions of PHY layer are listed below:

- WirelessMAN-SC: corresponds to the single-carrier version, designed to support LOS operation in the 10 to 66 $\mathrm{GHz}$ frequency range. The goal is to provide flexibility 
in LOS operation scenarios, in terms of planning, cost, services and capacity;

- WirelessMAN-SCa: this is the single-carrier solution for NLOS operation in frequencies below $11 \mathrm{GHz}$. The frame structure is designed to be robust against multipath fading. Furthermore, it supports channel estimation and equalization, adaptive modulation, multiple error correcting coding schemes, adaptive antennas, transmission diversity, power control and Automatic Repeat Request (ARQ);

- WirelessMAN-OFDM: designed to support NLOS operation in frequencies below $11 \mathrm{GHz}$, based on Orthogonal Frequency Division Multiplexing (OFDM), which consists of a multicarrier modulation scheme. This version extends the functionalities of version WirelessMAN-SCa, to support Mesh topology and subchannelization on the uplink, thus providing advanced resources for coverage optimization;

- WirelessMAN-OFDMA: this version supports NLOS operation in frequencies below $11 \mathrm{GHz}$, based on a Orthogonal Frequency Division Multiple Access (OFDMA), which consists of an extension of OFDM technique to allow multiple users access a shared channel. It consists of many of WirelesMAN-SCa functionalities, including support to subchannelization on uplink and downlink;

- WirelessHUMAN: due to the support to functionalities for operation in license-exempt frequencies, this version is named "High-speed Unlicensed Metropolitan Area Network" (HUMAN). It can operate at frequencies between 5 and $6 \mathrm{GHz}$, based on a flexible channelization scheme, which includes 10 and $20 \mathrm{MHz}$ channels, with $5 \mathrm{GHz}$ spacing. However, the channelization scheme to be adopted in particular deployment will depend on regulatory aspects. It is worth noting that this version applies to SCa, OFDM and OFDMA versions of PHY layer.

As one can see, the IEEE 802.16 standard is quite flexible in terms of operation frequency, supporting both license and license-exempt bands.

\section{NLOS Operation of IEEE 802.16 Based Systems}

Line of sight operation is often defined in terms of Fresnel zones [3]. It is shown that the diffraction in radio propagation is minimized if there is no obstacle within the first Fresnel zone, which concentrates most part of wave energy. In a real world deployment scenario, this condition can be accomplished by increasing antenna height.

Since LOS operation imposes severe constraints on the deployment of any wireless network, acceptable system performance under NLOS propagation becomes a major requirement to enable fast network expansion. The first step to enable NLOS propagation is to reduce the carrier frequency below $11 \mathrm{GHz}$, in order to increase wavelength, thus enhancing radio signal propagation. Furthermore, multipath becomes significant in lower frequencies, which can increase reception performance if appropriate techniques are adopted.

Besides operating at lower frequencies, a set of key functionalities must be implemented at the MAC and PHY layers in
Transmitter
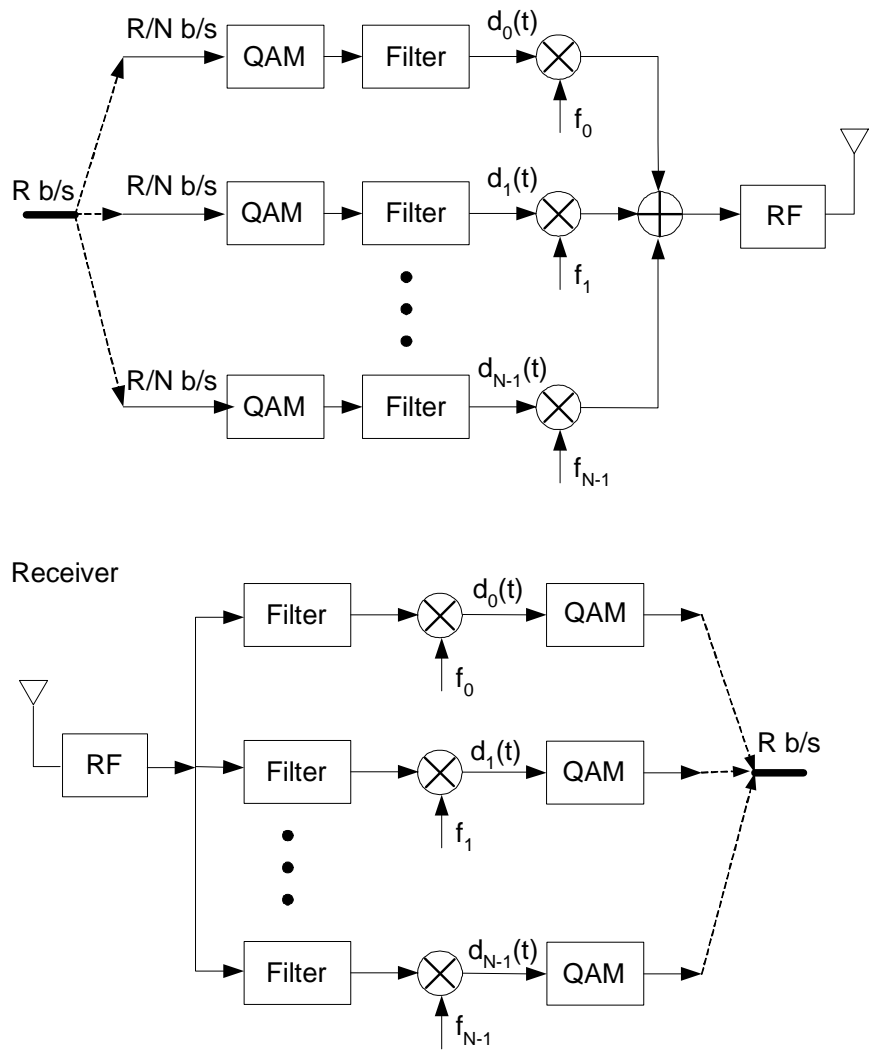

Fig. 3. OFDM implemented via bank of filters.

order to support NLOS operation in real world scenarios. The major functionalities specified in the IEEE 802.16 standard that support NLOS operation are described in the subsequent subsections.

1) OFDM Technique: The Orthogonal Frequency Division Multiplexing (OFDM) is a key technique to enable NLOS operation of WiMAX technology, due to the higher multipath robustness achieved at reception. OFDM operation consists of multiplexing information on multiple narrowband subchannels [8], modulated by a set of orthogonal subcarriers. This multicarrier scheme can be modeled as a bank of filters, as depicted in Figure 3, with each branch corresponding to a subchannel. In this approach, a data stream is transmitted at a rate of $R$ bps and is multiplexed on $N$ subchannels. Thus, the information rate at each filter branch is reduced by a factor of $N$, leading to a transmission rate of $R / N$ bps over each subcarrier.

The first benefit that arises from the transmission over narrowband subcarriers is the significant complexity reduction of channel equalization algorithms. Figure 4(a) illustrates the radio channel distortion over a wideband single-carrier transmission system. In Figure 4(b), a wideband transmission system is composed of multiple narrowband subcarriers, which are uniformly attenuated due to radio channel distortion. By comparing the effects of radio channel distortion in Figure 4, it becomes clear that equalization tends to be far less complex in radio transmission systems based on narrowband subcarriers, since it reduces to a simple gain recovery (amplification) 


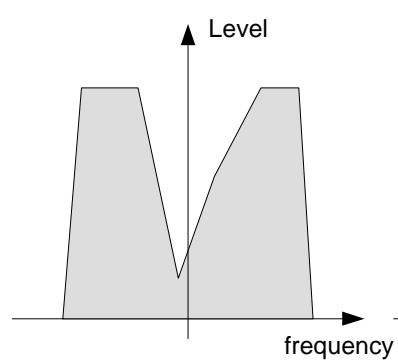

(a)

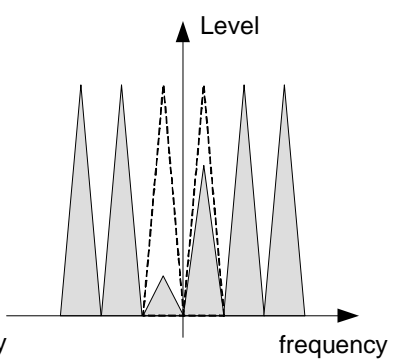

(b)
Fig. 4. Radio channel distortion in wideband single-carrier and multi-carrier systems: (a) single-carrier transmission system (b) multi-carrier transmission system.

procedure per subcarrier.

The OFDM scheme specified in the IEEE 802.16 standard is shown in Figure 5. The symbol structure is composed of a guard interval $\left(T_{g}\right)$ and the useful symbol interval $\left(T_{b}\right)$, with the resulting symbol duration equal to $T_{s}$, as depicted in Figure 5(a). The last $T_{g}$ portion of the useful symbol, named Cyclic Prefix (CP), in continuously copied on to the guard time portion. The adoption of Cyclic Prefix increases robustness against multipath fading, as well as the tolerance for symbol time synchronization errors. In fact, $\mathrm{CP}$ contributes to preserve subcarriers orthogonality.

In Figure 5(c), the different types of subcarriers are illustrated. The pilot subcarrier does not carry data or signaling information and allows the system to estimate key attributes for equalization and power control mechanisms (e.g., channel estimation and carrier-to-interference-and-noise-ratio). The DC subcarriers allow the inclusion of guard bands between groups of subcarriers, while the data subcarriers are employed for the data bits transmission.

The OFDM transmitter waveform $s_{a}(t)$, with no $\mathrm{CP}$, in defined as follows:

$$
s_{a}(t)=\sum_{k=0}^{N-1} a(k) e^{j 2 \pi \Delta f k t} \quad 0<t \leq T
$$

where $T_{s}=1 / \Delta f=N / W$.

A discrete-domain representation for $s(t)$ can be obtained by sampling st at rate $T_{s} / N$, such that

$$
s[n]=s_{a}\left(\frac{n T_{s}}{N}\right)=\sum_{k=0}^{N-1} a(k) e^{j 2 \pi \Delta f n k \frac{T_{s}}{N}} \quad 0<t \leq T_{s} .
$$

However, in order to achieve orthogonality, it is required that $\Delta f T_{s}=1$, thus resulting in (3) for the discrete-time OFDM transmitter symbol:

$$
s[n]=\sum_{k=0}^{N-1} a(k) e^{j 2 \pi n \frac{k}{N}}=\operatorname{IDFT}\{a(k)\} \quad 0<t \leq T_{s}
$$

where IDFT is the Inverse Discrete Fourier Transform. Currently, IDFT is efficiently implemented via IFFT (Inverse Fast Fourier Transform) algorithms. This approach leads to the discrete-time OFDM model, illustrated in Figure 6, which allows lower cost implementation via modern digital signal processing devices.

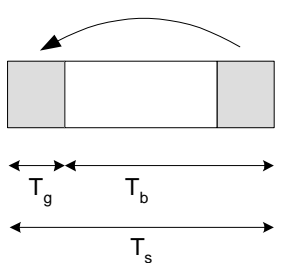

(a)

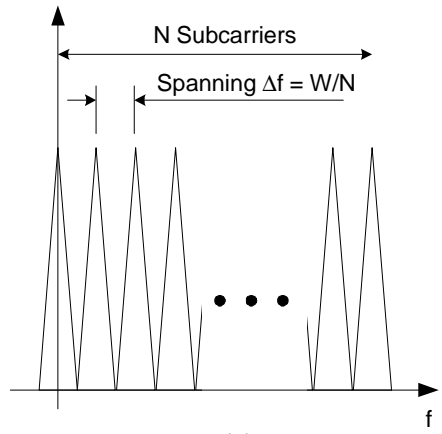

(b)

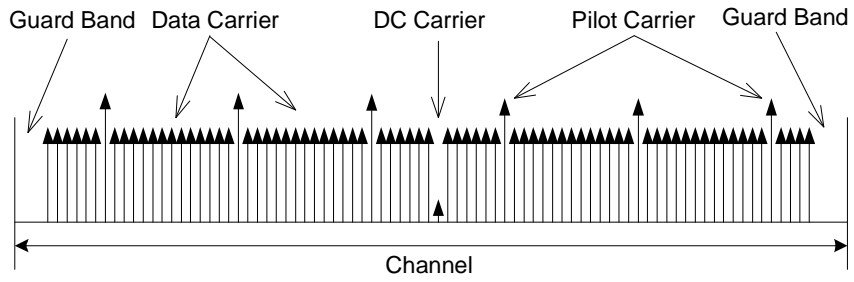

(c)

Fig. 5. OFDM technique in WiMAX technology: (a) symbol structure; (b) orthogonal subcarriers; (c) data, DC and pilot subcarriers.

Transmitter
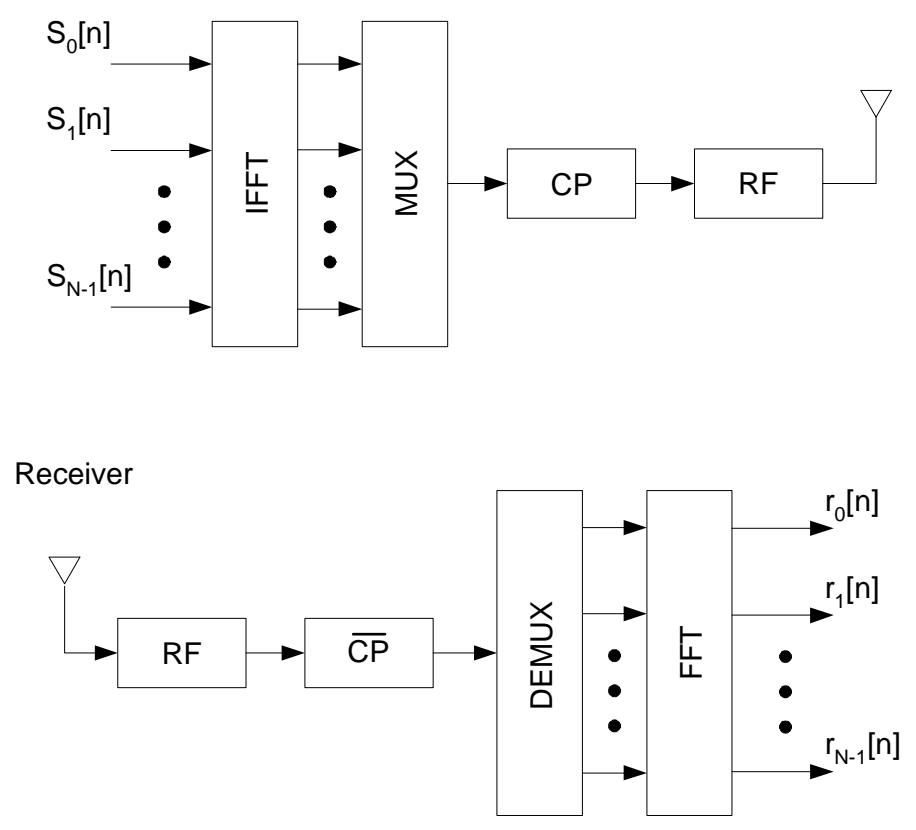

Fig. 6. Discrete-time OFDM model. 
$\mathrm{P}$

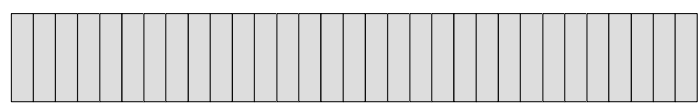

$\mathrm{P} / 4$

$\mathrm{P}$

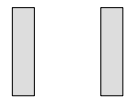

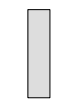

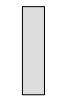

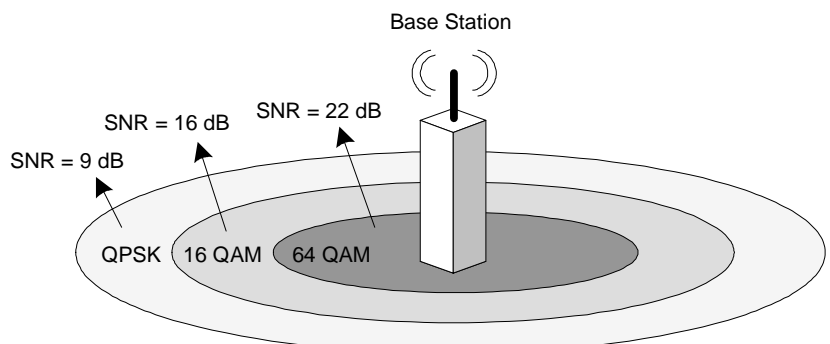

Fig. 7. Subchannelization scheme in WiMAX technology.

2) Subchannelization: Most wireless networks are subject to coverage unbalance between uplink and downlink. In fact, subscriber stations are often submitted to cost, physical and resource availability constraints (e.g., maximum antenna height, power consumption, maximum transmission power). Depending on transmission power constraints of the subscriber station, the system coverage is limited by the uplink coverage, thus causing the link unbalance problem.

In order to enhance uplink coverage, a subchannelization technique is specified in the IEEE 802.16 standard, for the OFDM version, illustrated in Figure 7. The SS transmission power is limited to $25 \%$ of the maximum BS transmission power. In order to increase uplink coverage, a subset of one forth of the available subchannels is selected for transmission, thus allowing the transmission power to be concentrated in a narrower frequency spectrum. By adopting this procedure, the resulting transmission power on the selected subchannels can be increased by a factor of 4 , which corresponds to the link balance condition. The price to be paid for coverage enhancement, however, is the reduction of available uplink bandwidth by a factor of 4 .

It is worth mentioning that subchannelization is also specified for downlink in the IEEE 802.16, but only for OFDMA PHY. In this approach, far away subscribers can be assigned subchannels with higher power, while nearby subscribers can be assigned subchannels with lower power, resulting in link budget and capacity improvement. This procedure also improves frequency reuse, due to partial reuse of channels and the possibility of sectors/cells to be assigned non-overlapping sets of subcarriers. Again, throughput reduction is the major drawback to be considered when adopting this approach.

3) Adaptive Modulation: In addition to OFDM multiplexing, adaptive modulation is adopted in the IEEE 802.16 standard. Depending on the signal-to-noise ratio $(S N R)$ at the receiver, the SS and the BS negotiate the most appropriate modulation scheme, among the available options (BPSK, QPSK, 16 QAM and 64 QAM), as illustrated in Figure 8. This approach maximizes throughput and connectivity within a cell, as it allows the system to switch between high performance modulation scheme (64-QAM) and high robustness modulation scheme (BPSK) schemes, as the distance between the BS to the SS varies. This approach has already been adopted in Wi-Fi technology [1]. Table I lists the adopted modulation and coding schemes defined in the IEEE 802.16 standard, along with the required $S N R$.

Fig. 8. Adaptive modulation scheme in WiMAX technology.

TABLE I

REQUIRED $S N R$ AT THE RECEIVER FOR EACH MODULATION SCHEME ADOPTED IN THE IEEE 802.16 STANDARD.

\begin{tabular}{|c|c|c|}
\hline Modulation Scheme & Coding Rate & $S N R(\mathrm{~dB})$ \\
\hline \hline BPSK & $1 / 2$ & 6.4 \\
\hline QPSK & $1 / 2$ & 9.4 \\
& $3 / 4$ & 11.2 \\
\hline 16-QAM & $1 / 2$ & 16.4 \\
& $3 / 4$ & 18.2 \\
\hline 64-QAM & $2 / 3$ & 22.7 \\
& $3 / 4$ & 24.4 \\
\hline
\end{tabular}

\section{Propagation Models}

Radio propagation loss estimation plays a key role in wireless network planning, as it is one of the factors that determine the sinal coverage. The maximum propagation loss tolerated by the system in a specific scenario is determined according to the well-known link budget calculation, which is represented by

$$
P_{r, \min }=P_{t}+G_{t}-L_{t}
$$

where $P_{r, \min }$ is the receiver sensitivity, $P_{t}$ is the transmission power, $G_{t}$ is the total system gain, and $L_{t}$ is the total system loss.

The total system loss corresponds to the sum of all losses in the system, including radio propagation loss $L$, cable losses and fading margins. On the other hand, the total gain comprises all the system contributions to enhance signal level at reception, including antenna and diversity gains.

The receiver sensitivity $P_{r, \text { min }}$ in $\mathrm{dBm}$ is given by

$$
P_{r, \min }=S N R_{r x}+10 \log (W)+F+N_{0}
$$

where $S N R_{r x}$ is the required signal-to-noise ratio in $\mathrm{dB}, W$ is the effective channel bandwidth in $\mathrm{Hz}, F$ is the noise figure in $\mathrm{dB}$, and $N_{0}=10 \log \left(k T / 10^{-3}\right)$ is the thermal noise level in $\mathrm{dBm}$, with $k=1.38 \times 10^{-23} \mathrm{~J} / \mathrm{K}$ (Boltzmann's constant) and $T$ being the temperature in Kelvin.

Several propagation models have already been proposed for a wide range of scenarios of wireless communications [9][10][11]. Considering the flexibility of WiMAX regarding the operation frequency, the present paper is focused on propagation models that provide acceptable accuracy for NLOS scenarios and operation frequency up to $6 \mathrm{GHz}$. For comparison purposes, LOS model is also considered in the 
TABLE II

SUI MODEL PARAMETERS.

\begin{tabular}{|c|c|c|c|}
\hline Model Parameter & Terrain Type A & Terrain Type B & Terrain Type C \\
\hline \hline $\mathrm{a}$ & 4.6 & 4 & 3.6 \\
\hline $\mathrm{b}$ & 0.0075 & 0.0065 & 0.005 \\
\hline $\mathrm{c}$ & 12.6 & 17.1 & 20 \\
\hline
\end{tabular}

analysis, which consists of the free space loss in $\mathrm{dB} L_{0}$, as described by

$$
L_{0}=32.45+20 \log \left(f_{c}\right)+20 \log (d)
$$

where $f_{c}$ is the operation frequency and $d$ is the distance in $\mathrm{km}$ between the subscriber station and the base station.

The COST 231 propagation model [12] is adopted in several real world applications, since it provides accurate estimates for NLOS propagation. Within COST 231 specifications, the Walfisch-Ikegami (Street-Canyon-SC) model is more appropriate for frequencies up to $6 \mathrm{GHz}$, since climate impacts can be neglected for frequencies between 2 and $6 \mathrm{GHz}$ [4]. The following expression describes the model

$$
L= \begin{cases}42.64+20 \log \left(f_{c}\right)+26 \log (d) & \text { if } d<d_{c} \\ 42.64+20 \log \left(f_{c}\right)+26 \log \left(d_{c}\right) & \\ +40 \log \left(\frac{d}{d_{c}}\right) & \text { if } d \geq d_{c}\end{cases}
$$

where $d_{c}=4 h_{t} h_{r} / \lambda$ is the breakpoint distance, with $h_{t}$ and $h_{r}$ being the transmit and receive antenna heights, respectively.

Recently, the Stanford University Interim (SUI) model has been proposed for Broadband Wireless Access (BWA) systems operating under NLOS condition [13]. It comprises a suburban path loss model and overcomes some limitations of Okumura-Hata model, which is not accurate for lower base station antenna heights and hilly or moderate-to-heavy wooded terrain. The radio propagation loss according to the SUI model is given by

$$
L=A+10 \gamma \log \left(\frac{d}{d_{0}}\right)+s \text { for } d<d_{0}
$$

where $A=20 \log \left(4 \pi d_{0} / \lambda\right), \lambda$ is the wavelength in meters, $\gamma=\left(a-b h_{b}+c / h_{b}\right)$ is the pathloss exponent, for base station antenna height $h_{b}$ between 10 and $80 \mathrm{~m}$ and reference distance $d_{0}$ of 100 meters. The constants $a, b$ and $c$ depends on the terrain type, as presented in Table II.

The variable $s$ in (8) represents the shadowing effect, modeled as a log-normal random variable with typical standard deviation between 8.2 and $10.6 \mathrm{~dB}$ [14]. Since the shadowing is already included in link budget calculations, it is not considered in SUI formula.

The SUI model is originally constrained to frequencies close to $2 \mathrm{GHz}$ and receiver antenna heights between 10 $\mathrm{m}$ and $80 \mathrm{~m}$. In order to overcome such limitations, an extrapolated version has been developed [15]. According to this extrapolated version, the path loss $L$ is now given by

$$
L=A+10 \gamma \log \left(\frac{d}{d_{0}}\right)+\Delta L_{f}+\Delta L_{h}+s \text { for } d<d_{0}
$$

where

$$
\Delta L_{f}=6 \log \left(\frac{f}{2000}\right)
$$

and

$$
\Delta L_{h}= \begin{cases}-10.8 \log \left(\frac{h}{2}\right), & \text { for terrain Types A and B } \\ -20 \log \left(\frac{h}{2}\right), & \text { for terrain Type C }\end{cases}
$$

where $f$ is the frequency in $\mathrm{MHz}$ and $h$ is the receive antenna height, between 2 and 10 meters.

\section{Coverage Prediction}

\section{A. Receiver sensitivity in WiMAX Technology}

The receiver sensitivity can be evaluated using (5), taking into account the particularities of the WiMAX technology and the scenario considered.

OFDM scheme in IEEE 802.16 standard does not allocate the entire channel bandwidth for information transmission. First of all, the FFT consumes part of channel bandwidth due to the sampling operation, which reduces effective bandwidth by a factor $F_{s} / B W$, where $F_{s}$ is the sampling frequency in $\mathrm{MHz}$ defined as

$$
F_{s}=\frac{\lfloor 8000 n B W\rfloor}{8000}
$$

where $n$ is the sampling factor and $B W$ is the channel bandwidth in $\mathrm{Hz}$.

Secondly, DC and guard band subcarriers transport no information, thus remaining $N_{\text {used }}$ subcarriers from the $N_{F F T}$ available subcarriers. Thus, the OFDM bandwidth efficiency is defined in the IEEE 802.16 standard as

$$
B W_{\text {efficiency }}=\frac{F_{s}}{B W} \frac{N_{u s e d}}{N_{F F T}}
$$

where $N_{\text {used }}$ is the number of subcarriers used, and $N_{F F T}$ is the length of FFT in OFDM PHY. Furthermore, the sampling frequency

The bandwidth efficiency is further reduced by the subchannelization scheme. In OFDM, up to 16 subchannels can be employed. If less than 16 subchannels are used, the transmission power is concentrated on a subset of available subcarriers. For instance, by using just one subcarrier, the power is concentrated in $1 / 16$ of the available subcarriers. Thus, the bandwidth is reduced by a factor of $N_{\text {subchannels }} / 16$, where $N_{\text {subchannels }}$ is limited to 16 . Insertion of this reduction factor in (13) results in

$$
B W_{\text {efficiency }}=\frac{F_{s}}{B W} \frac{N_{\text {used }}}{N_{F F T}} \frac{N_{\text {subchannels }}}{16} .
$$

The effective bandwidth $W$, required to evaluate 5), can be straightforwardly computed from (14)

$$
W=B W_{\text {efficiency }} B W=F_{s} \frac{N_{\text {used }}}{N_{F F T}} \frac{N_{\text {subchannels }}}{16} .
$$

In accordance to the OFDM specification in the IEEE 802.16 standard, the noise figure is equal to $5 \mathrm{~dB}$ added to an implementation margin of $7 \mathrm{~dB}$, thus resulting in effective noise figure $F=12 \mathrm{~dB}$. 
TABLE III

OFDM PHY PARAMETERS, ACCORDING TO THE IEEE 802.16 STANDARD.

\begin{tabular}{|c|l|}
\hline Parameter & OFDM value \\
\hline \hline$N_{\text {used }}$ & 192 \\
\hline$N_{F F T}$ & 256 \\
\hline$b_{m}$ & 1 (BPSK), 2(QPSK), 4 (16-QAM) and 6 (64-QAM) \\
\hline$c_{r}$ & $1 / 2,2 / 3$ and $3 / 4$ \\
\hline$G$ & $1 / 4,1 / 8,1 / 16$ and $1 / 32$ \\
\hline$n$ & $* 8 / 7$, for BW a multiple of $1.75 \mathrm{MHz}$ \\
& $* 86 / 75$, for BW a multiple of $1.5 \mathrm{MHz}$ \\
& $* 144 / 125$, for BW a multiple of $1.25 \mathrm{MHz}$ \\
& $* 316 / 275$, for BW a multiple of $2.75 \mathrm{MHz}$ \\
& $* 57 / 50$, for BW a multiple of $2.0 \mathrm{MHz}$ \\
& $* 8 / 7$, otherwise \\
\hline
\end{tabular}

Next, considering the temperature of $T=290 \mathrm{~K}$ results in a thermal noise of $N_{0}=-174 \mathrm{dBm}$, which is in fact adopted in most practical applications.

Finally, substituting (15) and the values for $N_{0}$ and $F$ into (5), the receiver sensitivity for OFDM physical layer is given by

$P_{r, \text { min }}=-102+S N R_{r x}+10 \log \left(\frac{F_{s} N_{\text {used }} N_{\text {subchannels }}}{16 N_{F F T}}\right)$.

In this expression, a $60 \mathrm{~dB}$ correction factor was added to account for frequency scale conversion from $\mathrm{Hz}$ to $\mathrm{MHz}$.

\section{B. Performance Analysis}

The maximum transmission data rate $R$ that can be achieved in OFDM PHY is defined in the IEEE 802.16 standard as

$$
R=\frac{N_{\text {used }} b_{m} c_{r}}{T_{s}}
$$

where $b_{m}$ is the number of bits per modulation symbol and $c_{r}$ is the coding rate. According to Figure 5(a), the symbol duration is $T_{s}$ is given by

$$
\begin{aligned}
T_{s} & =T_{g}+T_{b} \\
& =[G+1] T_{b}
\end{aligned}
$$

where $T_{b}=1 / \Delta f$, with the subcarrier spacing $\Delta f$ given by

$$
\Delta f=\frac{F_{s}}{N_{F F T}}
$$

and $G$ is the ratio $T_{g} / T_{b}$. In Table III, the values of the most relevant system parameters are defined, in accordance to the IEEE 802.16 standard.

\section{Coverage Prediction Evaluation}

In this work, coverage prediction is evaluated for licensed and license-exempt bands, in accordance to the current Brazilian regulatory rules [6]. We adopted coverage and bandwidth optimized system configurations. The resulting scenarios are described in Table IV.

The coverage prediction results for Scenario A are presented in Tables V and VI, and the following observations can be

\begin{tabular}{|c|c|c|}
\hline Scenario & Description & Parameters \\
\hline A & $\begin{array}{l}\text { Licensed } \\
\text { operation, } \\
\text { bandwidth } \\
\text { optimized, } \\
\text { outdoor }\end{array}$ & $\begin{array}{l}W: 1.75 \text { and } 7 \mathrm{MHz} \\
F_{c}: 3.5 \mathrm{GHz} \text { (licensed) } \\
\text { Modulations: QPSK and 64-QAM } \\
\text { Coding rates: } 1 / 2,2 / 3 \text { and } 3 / 4 \\
N_{\text {subchannels }}: 16 \text { (no subchannel.) } \\
\text { Tx Power: } 22 \mathrm{dBm} \\
\text { Antennas gain: } 3 \mathrm{dBi}(\mathrm{BS}), 15 \mathrm{dBi}(\mathrm{SS})\end{array}$ \\
\hline B & $\begin{array}{l}\text { Licensed } \\
\text { operation, } \\
\text { coverage } \\
\text { optimized, } \\
\text { outdoor }\end{array}$ & $\begin{array}{l}W: 1.75 \text { and } 7 \mathrm{MHz} \\
F_{c}: 3.5 \mathrm{GHz} \text { (licensed) } \\
\text { Modulations: QPSK and 64-QAM } \\
\text { Coding rates: } 1 / 2,2 / 3 \text { and } 3 / 4 \\
N_{\text {subchannels }}: 4 \\
\text { Tx Power: } 22 \mathrm{dBm} \\
\text { Antennas gain: } 3 \mathrm{dBi}(\mathrm{BS}), 15 \mathrm{dBi}(\mathrm{SS})\end{array}$ \\
\hline $\mathrm{C}$ & $\begin{array}{l}\text { License-exempt } \\
\text { operation, } \\
\text { bandwidth } \\
\text { optimized }\end{array}$ & $\begin{array}{l}W: 10 \text { and } 20 \mathrm{MHz} \\
F_{c}: 5.8 \mathrm{GHz} \text { (license-exempt) } \\
\text { Modulations: QPSK and 64-QAM } \\
\text { Coding rates: } 1 / 2,2 / 3 \text { and } 3 / 4 \\
N_{\text {subchannels }}: 16 \text { (no subchannel.) } \\
\text { Tx Power: } 20 \mathrm{dBm} \\
\text { Antennas gain: } 5 \mathrm{dBi}(\mathrm{BS}), 3 \mathrm{dBi}(\mathrm{SS})\end{array}$ \\
\hline $\mathrm{D}$ & $\begin{array}{l}\text { License-exempt } \\
\text { operation, } \\
\text { coverage } \\
\text { optimized }\end{array}$ & $\begin{array}{l}W: 10 \text { and } 20 \mathrm{MHz} \\
F_{c}: 5.8 \mathrm{GHz} \text { (license-exempt) } \\
\text { Modulations: QPSK and 64-QAM } \\
\text { Coding rates: } 1 / 2,2 / 3 \text { and } 3 / 4 \\
N_{\text {subchannels }}: 2 \\
\text { Tx Power: } 20 \mathrm{dBm} \\
\text { Antennas gain: } 5 \mathrm{dBi}(\mathrm{BS}), 3 \mathrm{dBi}(\mathrm{SS})\end{array}$ \\
\hline
\end{tabular}
made:
TABLE IV

COVERAGE PREDICTION FOR OUTDOOR SCENARIOS

TABLE V

ESTIMATED UPLINK COVERAGE RADIUS FOR SCENARIO A, IN KM.

\begin{tabular}{|c||c|c|c|c||c|c|c|c|}
\hline \multicolumn{1}{|c||}{$\begin{array}{c}\text { Propagation } \\
\text { Model }\end{array}$} & \multicolumn{4}{c||}{$\mathbf{1 . 7 5} \mathbf{~ M H z}$} & \multicolumn{4}{c|}{$7 \mathbf{~ M H z}$} \\
\cline { 2 - 9 } & QPSK & \multicolumn{6}{c|}{ 64-QAM } & \multicolumn{2}{|c|}{ QPSK } & 64-QAM \\
\cline { 2 - 9 } & $\mathbf{1 / 2}$ & $\mathbf{3 / 4}$ & $\mathbf{2 / 3}$ & $\mathbf{3 / 4}$ & $\mathbf{1 / 2}$ & $\mathbf{3 / 4}$ & $\mathbf{2 / 3}$ & $\mathbf{3 / 4}$ \\
\hline \hline LOS & 9.4 & 7.7 & 2.0 & 1.7 & 4.7 & 3.8 & 2.1 & 1.7 \\
\hline W-I (SC) & 2.3 & 1.9 & 0.7 & 0.6 & 1.3 & 1.1 & 0.7 & 0.6 \\
\hline SUI C & 0.8 & 0.8 & 0.4 & 0.4 & 0.6 & 0.5 & 0.4 & 0.4 \\
\hline SUI B & 0.7 & 0.7 & 0.4 & 0.3 & 0.5 & 0.5 & 0.4 & 0.3 \\
\hline SUI A & 0.6 & 0.6 & 0.3 & 0.3 & 0.5 & 0.4 & 0.3 & 0.3 \\
\hline
\end{tabular}

- Operation in terrain types defined in SUI model provides the smallest coverage, with maximum radius below $1 \mathrm{~km}$ for the uplink and $1.5 \mathrm{~km}$ for downlink;

- The links are not balanced, since uplink coverage radius is always smaller than the downlink coverage radius;

- The highest performance modulation (64 QAM) and coding schemes provide the worst coverage;

- Increasing the channel bandwidth $B W$ leads to a coverage reduction, which can be explained by the increase of effective channel bandwidth $W$, as per (15), which consequently degrades receiver sensitivity, according to (16).

The impact of bandwidth $B W$ on coverage is in accordance to OFDM characteristics. In fact, by increasing $B W$ and 
TABLE VI

ESTIMATED DOWNLINK COVERAGE RADIUS FOR SCENARIO A, IN KM.

\begin{tabular}{|c||c|c|c|c||c|c|c|c|}
\hline \multicolumn{1}{|c||}{\begin{tabular}{c} 
Propagation \\
\multirow{2}{*}{ Model }
\end{tabular}} & \multicolumn{4}{c||}{$\mathbf{1 . 7 5} \mathbf{~ M H z}$} & \multicolumn{4}{c|}{ 7 MHz } \\
\cline { 2 - 9 } & \multicolumn{2}{c|}{ QPSK } & \multicolumn{1}{c|}{ 64-QAM } & \multicolumn{2}{c|}{ QPSK } & \multicolumn{2}{|c|}{ 64-QAM } \\
\cline { 2 - 9 } & $\mathbf{1 / 2}$ & $\mathbf{3 / 4}$ & $\mathbf{2 / 3}$ & $\mathbf{3 / 4}$ & $\mathbf{1 / 2}$ & $\mathbf{3 / 4}$ & $\mathbf{2 / 3}$ & $\mathbf{3 / 4}$ \\
\hline \hline LOS & 33.5 & 27.3 & 7.3 & 6.0 & 16.8 & 13.6 & 7.5 & 6.1 \\
\hline W-I (SC) & 6.0 & 5.2 & 1.9 & 1.6 & 3.5 & 3.0 & 1.9 & 1.6 \\
\hline SUI C & 1.6 & 1.4 & 0.7 & 0.7 & 1.1 & 1.0 & 0.8 & 0.7 \\
\hline SUI B & 1.3 & 1.2 & 0.7 & 0.6 & 1.0 & 0.9 & 0.7 & 0.6 \\
\hline SUI A & 1.1 & 1.0 & 0.6 & 0.5 & 0.8 & 0.7 & 0.6 & 0.5 \\
\hline
\end{tabular}

TABLE VII

ESTIMATED UPLINK COVERAGE RADIUS FOR SCENARIO B, IN KM.

\begin{tabular}{|c|c|c|c|c|c|c|c|c|}
\hline \multirow{3}{*}{$\begin{array}{c}\text { Propagation } \\
\text { Model }\end{array}$} & \multicolumn{4}{|c|}{$1.75 \mathrm{MHz}$} & \multicolumn{4}{|c|}{$7 \mathrm{MHz}$} \\
\hline & \multicolumn{2}{|c|}{ QPSK } & \multicolumn{2}{|c|}{ 64-QAM } & \multicolumn{2}{|c|}{ QPSK } & \multicolumn{2}{|c|}{ 64-QAM } \\
\hline & $1 / 2$ & $3 / 4$ & $2 / 3$ & $3 / 4$ & $1 / 2$ & $3 / 4$ & $2 / 3$ & $3 / 4$ \\
\hline LOS & 33.7 & 27.4 & 7.3 & 6.0 & 16.8 & 13.7 & 7.5 & 6.1 \\
\hline W-I (SC) & 6.1 & 5.2 & 1.9 & 1.6 & 3.6 & 3.0 & 1.9 & 1.6 \\
\hline SUI C & 1.6 & 1.4 & 0.7 & 0.7 & 1.1 & 1.0 & 0.8 & 0.7 \\
\hline SUI B & 1.3 & 1.2 & 0.6 & 0.6 & 1.0 & 0.9 & 0.7 & $\overline{0.6}$ \\
\hline SUI A & 1.1 & 1.0 & 0.6 & 0.5 & 0.8 & 0.7 & 0.6 & 0.5 \\
\hline
\end{tabular}

keeping the same number of used subcarriers, the subcarrier bandwidth increases, which reduces the effectiveness of equalization and coding schemes, thus degrading the overall OFDM receiver performance.

In Tables VII and VIII, coverage prediction for Scenario B is presented. In order to optimize uplink coverage, subchannelization is implemented $\left(N_{\text {subchannels }}=1\right)$, thus resulting in a virtually balanced link scenario.

The estimated maximum uplink data rate, for Scenarios A and $\mathrm{B}$, is described in Table IX. Due to subchannelization, the maximum data rate that can be achieved in Scenario B is significantly lower than that for Scenario A (about 1/16), corresponding to the cost of increasing uplink coverage. It can also be noticed that the maximum uplink data rates are 16.9

TABLE VIII

ESTIMATED DOWNLINK COVERAGE RADIUS FOR SCENARIO B, IN KM.

\begin{tabular}{|c|c|c|c|c|c|c|c|c|}
\hline \multirow{3}{*}{$\begin{array}{c}\text { Propagation } \\
\text { Model }\end{array}$} & \multicolumn{4}{|c|}{$1.75 \mathrm{MHz}$} & \multicolumn{4}{|c|}{$7 \mathrm{MHz}$} \\
\hline & \multicolumn{2}{|c|}{ QPSK } & \multicolumn{2}{|c|}{ 64-QAM } & \multicolumn{2}{|c|}{ QPSK } & \multicolumn{2}{|c|}{ 64-QAM } \\
\hline & $1 / 2$ & $3 / 4$ & $2 / 3$ & $3 / 4$ & $1 / 2$ & $3 / 4$ & $2 / 3$ & $3 / 4$ \\
\hline LOS & 37.6 & 30.6 & 8.1 & 6.7 & 18.8 & 15.3 & 8.4 & 6.8 \\
\hline W-I (SC) & 6.6 & 5.6 & 2.0 & 1.7 & 3.9 & 3.3 & 2.1 & 1.8 \\
\hline SUI C & 1.6 & 1.5 & 0.8 & 0.7 & 1.2 & 1.1 & 0.8 & 0.7 \\
\hline SUI B & 1.4 & 1.3 & 0.7 & 0.6 & 1.0 & 0.9 & 0.7 & 0.6 \\
\hline SUI A & 1.1 & 1.0 & 0.6 & 0.5 & 0.8 & 0.8 & 0.6 & 0.5 \\
\hline
\end{tabular}

TABLE IX

ESTIMATED MAXIMUM UPLINK DATA RATE IN MBPS, FOR LICENSED OPERATION

\begin{tabular}{|c||c|c|c|c||c|c|c|c|}
\hline \multirow{2}{*}{ Scenario } & \multicolumn{4}{c||}{$\mathbf{1 . 7 5} \mathbf{~ M H z}$} & \multicolumn{4}{c|}{$\mathbf{7 ~ M H z}$} \\
\cline { 2 - 9 } & \multicolumn{2}{|c|}{ QPSK } & \multicolumn{1}{|c|}{ 64-QAM } & \multicolumn{2}{|c|}{ QPSK } & 64-QAM \\
\cline { 2 - 9 } & $\mathbf{1 / 2}$ & $\mathbf{3 / 4}$ & $\mathbf{2 / 3}$ & $\mathbf{3 / 4}$ & $\mathbf{1 / 2}$ & $\mathbf{3 / 4}$ & $\mathbf{2 / 3}$ & $\mathbf{3 / 4}$ \\
\hline \hline $\mathrm{A}$ & 1.4 & 2.1 & 5.6 & 6.4 & 5.6 & 8.5 & 22.6 & 25.4 \\
\hline $\mathrm{B}$ & 0.09 & 0.13 & 0.4 & 0.4 & 0.35 & 0.53 & 1.41 & 1.59 \\
\hline
\end{tabular}

TABLE $X$

ESTIMATED UPNLINK COVERAGE RADIUS FOR SCENARIO C, IN KM.

\begin{tabular}{|c||c|c|c|c||c|c|c|c|}
\hline \multicolumn{1}{|c||}{$\begin{array}{c}\text { Propagation } \\
\text { Model }\end{array}$} & \multicolumn{4}{c||}{$\mathbf{1 0} \mathbf{~ M H z}$} & \multicolumn{4}{c|}{$\mathbf{2 0 ~ M H z}$} \\
\cline { 2 - 9 } & QPSK & \multicolumn{1}{c|}{ 64-QAM } & \multicolumn{2}{|c|}{ QPSK } & \multicolumn{2}{|c|}{ 64-QAM } \\
\cline { 2 - 9 } & $\mathbf{1 / 2}$ & $\mathbf{3 / 4}$ & $\mathbf{2 / 3}$ & $\mathbf{3 / 4}$ & $\mathbf{1 / 2}$ & $\mathbf{3 / 4}$ & $\mathbf{2 / 3}$ & $\mathbf{3 / 4}$ \\
\hline \hline LOS & 1.2 & 1.0 & 0.3 & 0.2 & 0.8 & 0.7 & 0.2 & 0.1 \\
\hline W-I (SC) & 0.5 & 0.4 & 0.1 & 0.1 & 0.4 & 0.3 & 0.1 & 0.1 \\
\hline SUI C & 0.3 & 0.3 & 0.1 & 0.1 & 0.2 & 0.2 & 0.1 & 0.1 \\
\hline SUI B & 0.3 & 0.3 & 0.1 & 0.1 & 0.2 & 0.2 & 0.1 & 0.1 \\
\hline SUI A & 0.2 & 0.2 & 0.1 & 0.1 & 0.2 & 0.2 & 0.1 & 0.1 \\
\hline
\end{tabular}

TABLE XI

ESTIMATED DOWNLINK COVERAGE RADIUS FOR SCENARIO C, IN KM.

\begin{tabular}{|c||c|c|c|c||c|c|c|c|}
\hline \multicolumn{1}{|c||}{\multirow{2}{*}{$\begin{array}{c}\text { Propagation } \\
\text { Model }\end{array}$}} & \multicolumn{4}{c||}{$\mathbf{1 0}$ MHz } & \multicolumn{4}{c|}{$\mathbf{2 0}$ MHz } \\
\cline { 2 - 9 } & QPSK & \multicolumn{1}{c|}{ 64-QAM } & \multicolumn{2}{|c|}{ QPSK } & \multicolumn{2}{|c|}{ 64-QAM } \\
\cline { 2 - 9 } & $\mathbf{1 / 2}$ & $\mathbf{3 / 4}$ & $\mathbf{2 / 3}$ & $\mathbf{3 / 4}$ & $\mathbf{1 / 2}$ & $\mathbf{3 / 4}$ & $\mathbf{2 / 3}$ & $\mathbf{3 / 4}$ \\
\hline \hline LOS & 3.8 & 3.1 & 0.8 & 0.7 & 2.7 & 2.2 & 0.6 & 0.5 \\
\hline W-I (SC) & 1.1 & 1.0 & 0.3 & 0.3 & 0.9 & 0.9 & 0.3 & 0.2 \\
\hline SUI C & 0.5 & 0.5 & 0.2 & 0.2 & 0.4 & 0.4 & 0.2 & 0.2 \\
\hline SUI B & 0.5 & 0.4 & 0.2 & 0.2 & 0.4 & 0.4 & 0.2 & 0.2 \\
\hline SUI A & 0.5 & 0.4 & 0.2 & 0.2 & 0.3 & 0.3 & 0.2 & 0.2 \\
\hline
\end{tabular}

Mbps for Scenario A and 1.6 Mbps for Scenario B, which were achieved for $7 \mathrm{MHz}$ channel bandwidth and 64 QAM modulation with $3 / 4$ coding rate. In Scenario B, this data rate is achievable for coverage radius about $6.1 \mathrm{~km}$ in LOS condition and $500 \mathrm{~m}$ for suburban environments (SUI model).

In the license-exempt operation scenarios (C and D), the coverage is severely degraded when compared to Scenarios A and $\mathrm{B}$, due to higher operation frequency $(5.8 \mathrm{GHz})$ and larger channel bandwidth (10 and $20 \mathrm{MHz}$ ). Tables $\mathrm{X}$ and XI show the coverage prediction results for Scenario C. The results show that the maximum uplink coverage radius is below 300 $\mathrm{m}$, for SUI model, and $500 \mathrm{~m}$, for Walfisch-Ikegami model. Even in LOS operation, the uplink radius is limited to $1.2 \mathrm{~km}$. Furthermore, uplink and downlink coverage is not balanced in this scenario.

Tables XII and XIII present the estimates for uplink and downlink coverage in Scenario D. Due to subchannelization ( $\left.N_{\text {subchannels }}=2\right)$, the uplink coverage is increased when compared to that for Scenario $\mathrm{C}$, thus mitigating link unbalance condition.

In Table XIV, the maximum uplink data rate for Scenarios $\mathrm{C}$ and D are compared. Due to subchannelization $(1 / 8)$, the maximum data rate in Scenario $\mathrm{D}$ is about $12,5 \%$ of data

TABLE XII

ESTIMATED UPLINK COVERAGE RADIUS FOR SCENARIO D, IN KM.

\begin{tabular}{|c||c|c|c|c||c|c|c|c|}
\hline \multicolumn{1}{|c||}{$\begin{array}{c}\text { Propagation } \\
\text { Model }\end{array}$} & \multicolumn{4}{c||}{$\mathbf{1 0} \mathbf{M H z}$} & \multicolumn{4}{c|}{$\mathbf{2 0} \mathbf{M H z}$} \\
\cline { 2 - 9 } & \multicolumn{2}{|c|}{ QPSK } & \multicolumn{2}{c|}{ 64-QAM } & \multicolumn{2}{c|}{ QPSK } & \multicolumn{2}{c|}{ 64-QAM } \\
\cline { 2 - 9 } & $\mathbf{1 / 2}$ & $\mathbf{3 / 4}$ & $\mathbf{2 / 3}$ & $\mathbf{3 / 4}$ & $\mathbf{1 / 2}$ & $\mathbf{3 / 4}$ & $\mathbf{2 / 3}$ & $\mathbf{3 / 4}$ \\
\hline \hline LOS & 3.4 & 2.7 & 0.7 & 0.6 & 2.4 & 1.9 & 0.5 & 0.4 \\
\hline W-I (SC) & 1.0 & 0.9 & 0.3 & 0.3 & 0.8 & 0.7 & 0.2 & 0.2 \\
\hline SUI C & 0.5 & 0.4 & 0.2 & 0.2 & 0.4 & 0.4 & 0.2 & 0.2 \\
\hline SUI B & 0.4 & 0.4 & 0.2 & 0.2 & 0.4 & 0.3 & 0.2 & 0.2 \\
\hline SUI A & 0.4 & 0.3 & 0.2 & 0.2 & 0.3 & 0.3 & 0.2 & 0.2 \\
\hline
\end{tabular}


TABLE XIII

ESTIMATED DOWNLINK COVERAGE RADIUS FOR SCENARIO D, IN KM.

\begin{tabular}{|c||c|c|c|c||c|c|c|c|}
\hline \multicolumn{1}{|c||}{$\begin{array}{c}\text { Propagation } \\
\text { Model }\end{array}$} & \multicolumn{4}{c||}{$\mathbf{1 0} \mathbf{~ M H z}$} & \multicolumn{4}{c|}{$\mathbf{2 0 ~ M H z}$} \\
\cline { 2 - 9 } & QPSK & \multicolumn{1}{c||}{ 64-QAM } & \multicolumn{2}{|c|}{ QPSK } & 64-QAM \\
\cline { 2 - 9 } & $\mathbf{1 / 2}$ & $\mathbf{3 / 4}$ & $\mathbf{2 / 3}$ & $\mathbf{3 / 4}$ & $\mathbf{1 / 2}$ & $\mathbf{3 / 4}$ & $\mathbf{2 / 3}$ & $\mathbf{3 / 4}$ \\
\hline \hline LOS & 3.8 & 3.1 & 0.8 & 0.7 & 2.7 & 2.2 & 0.6 & 0.5 \\
\hline W-I (SC) & 1.1 & 1.0 & 0.3 & 0.3 & 0.9 & 0.7 & 0.3 & 0.2 \\
\hline SUI C & 0.5 & 0.5 & 0.2 & 0.2 & 0.4 & 0.4 & 0.2 & 0.2 \\
\hline SUI B & 0.5 & 0.4 & 0.2 & 0.2 & 0.4 & 0.4 & 0.2 & 0.2 \\
\hline SUI A & 0.4 & 0.4 & 0.2 & 0.2 & 0.3 & 0.3 & 0.2 & 0.2 \\
\hline
\end{tabular}

TABLE XIV

ESTIMATED MAXIMUM UPLINK DATA RATE IN MBPS, FOR LICENSE-EXEMPT OPERATION

\begin{tabular}{|c|c|c|c|c|c|c|c|c|}
\hline \multirow{3}{*}{ Scenario } & \multicolumn{4}{|c|}{$10 \mathrm{MHz}$} & \multicolumn{4}{|c|}{$20 \mathrm{MHz}$} \\
\hline & \multicolumn{2}{|c|}{ QPSK } & \multicolumn{2}{|c|}{ 64-QAM } & \multicolumn{2}{|c|}{ QPSK } & \multicolumn{2}{|c|}{ 64-QAM } \\
\hline & $1 / 2$ & $3 / 4$ & $2 / 3$ & $3 / 4$ & $1 / 2$ & $3 / 4$ & $2 / 3$ & $3 / 4$ \\
\hline $\mathrm{C}$ & 10.8 & 12.2 & 32.5 & 36.6 & 16.3 & 24.4 & 65.1 & 73.2 \\
\hline $\mathrm{D}$ & 1.36 & 1.52 & 4.1 & 4.6 & 2.03 & 3.05 & 8.13 & 9.15 \\
\hline
\end{tabular}

rates achieved in Scenario C. The maximum uplink data rates are about $73 \mathrm{Mbps}$ in Scenario C and 9 Mbps in Scenario D, which were achieved for $20 \mathrm{MHz}$ channel bandwidth, 64QAM modulation with $3 / 4$ coding rate, and coverage radius below $500 \mathrm{~m}$ for LOS condition and $200 \mathrm{~m}$ for suburban scenarios (SUI Model).

Comparing Tables IX and XIV, it can been noticed that operation in licensed bands $(3.5 \mathrm{GHz})$ provides better coverage but with lower throughput than in license-exempt bands (5.8 $\mathrm{GHz}$ ), due to the narrower channel bandwidth available for licensed operation.

\section{Conclusion}

This paper presented an overview of the major functionalities that support NLOS operation of WiMAX technology, in accordance to the IEEE 802.16-2004 standard, with focus on OFDM physical layer.

The WiMAX OFDM receiver sensitivity was analyzed, thus enabling coverage prediction based on the system parameters defined in the IEEE 802.16 standard, including subchannelization, modulation and codification schemes.

The current propagation models for broadband wireless, that support operation frequency above $2 \mathrm{GHz}$ and below 6 $\mathrm{GHz}$, were described and adopted for link budget calculation purposes. Then, coverage prediction was performed, based on licensed and license-exempt operation scenarios.

Finally, OFDM performance was evaluated, in terms of maximum uplink data transmission rate, which is degraded in coverage optimized system configurations, due to subchannelization. The scenarios were defined in accordance to the current Brazilian regulatory rules for radio-frequency spectrum utilization, for licensed and license-exempt bands.

\section{REFERENCES}

[1] IEEE 802.11b-1999, "IEEE standard for information technology telecommunications and information exchange between systems - local and metropolitan area networks - specific requirement. Part 11: wireless LAN medium access control (MAC) and Physical layer (PHY) specifications", 1999.

[2] IEEE 802.16-2004, "IEEE Standard for Local and metropolitan area networks Part 16: Air Interface for Fixed and Mobile Broadband Wireless Access Systems", Dec. 2005.

[3] M. D. Yacoub, Foundations of Mobile Radio Engineering, CRC Press, 1993.

[4] G. Plitsis, "Coverage Prediction of New Elements of Systems Beyond 3G: The IEEE 802.16 System as a Case Study", Communication Networks, Aachen University, Kopernikusstr. 16, D-52074 Aachen, Germany.

[5] "WiMAX's technology for LOS and NLOS environments", WiMAX Forum. [Online]. Available: www.wimaxforum.org.

[6] "Regulamento sobre equipamentos de radiocomunicação de radiação restrita, Resolução 365- ANATEL May 2004, Brazil.

[7] "Regulamento sobre condições de uso da faixa de freqüências de 3.5 GHz", Resolução 309- ANATEL. In Porthuguese. September 2002, Brazil.

[8] O. Edfors, M. Sandell, J-J V. de Beek, D. Landstrm, F. Sjberg, "An introduction to orthogonal frequency division multiplexing". [Online]. Available: http://courses.ece.uiuc.edu/ece559/spring02/ofdmtutorial.pdf.

[9] Y. Okumura, E. Ohmori, T. Kawano, and K. Fukua, "Field strength and its variability in UHF and VHF land-mobile radio service", Elec. Commun. Lab., vol. 16, no. 9, 1968.

[10] M. Hata, "Empirical formula for propagation loss in land mobile radio services", IEEE Trans. Veh. Technol., vol. 29, pp. 317-325, Aug. 1980.

[11] W.C. Jakes and D.O. Reudink, "Comparison of mobile radio transmission at UHF and X-band", IEEE Trans. Veh. Technol., vol. 16, pp. 10-13, Oct. 1967.

[12] Digital Mobile Radio Towards Future Generation System", COST 231 Final Report. [Online]. Available: http://www.lx.it.pt/cost231/final_report.htm.

[13] V. Erceg, et. al, "Channel Models for Fixed Wireless Applications", IEEE 802.16.3 Task Group Contributions 2001, Feb. 2001

[14] V. Erceg et. al, "An empirically based path loss model for wireless channels in suburban environments", IEEE Journal on Selected Areas on Communications, vol. 17, no. 7, pp. 1205-1211, July 1999.

[15] T.-S. Chu and L.J. Greenstein, "A quantification of link budget differences between the cellular and PCS bands", IEEE Trans. Veh. Technol., vol. 48 , no. 1 , pp. 60-65, Jan. 1999.

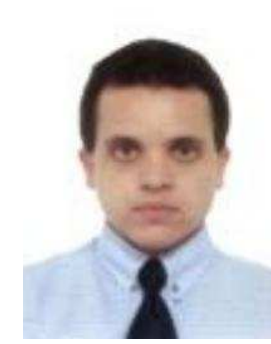

Fabricio Lira Figueiredo received the BS degree in electrical engineering from the Federal University of Pernambuco, in 1995. He received the MS degree in electrical engineering from the State University of Campinas, in 1999. He is currently a researcher at $\mathrm{CPqD}$ Telecom and IT Solutions, focused on advanced wireless networks. Current interests include ad hoc wireless networks, wireless local and metropolitan area networks, capacity an QoS in wireless networks, wireless sensor networks, cellular ing and speech recognition. networks, mobile applications, digital signal process-

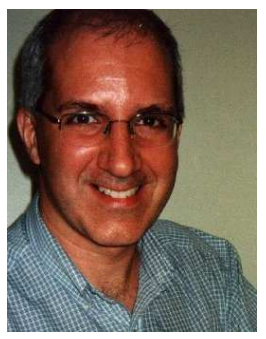

Paulo Cardieri received the B.S. degree from Mauá School of Engineering, Brazil, in 1987, the M.Sc. degree from the State University of Campinas, Brazil, in 1994, and the Ph.D. degree from Virginia Polytechnic Institute and State University, USA, all in electrical engineering. He is currently an assistant professor at the School of Electrical and Computer Engineering of UNICAMP. Prior to joining the faculty of UNICAMP, he was with the CPqD Foundation, Campinas, Brazil, where he was involved with research projects in several areas, including satellite and wireless communications. His current research interests include wireless ad hoc network, sensor network, and smart antennas. 